Health Inspection Service (Washington, DC, USA) before it can be returned to regular service. The entire production process must be thoroughly documented and is very tightly controlled.

We feel that the critical underlying focus should be on keeping the recombinant proteins out of the food supply regardless of what crop was used to make them. The crops should be grown under suitable containment conditions - rather than arbitrarily ruling out specific vehicles. Also, the risk associated with each protein should be reviewed on the basis of the properties of that specific protein, not on the basis of the production system used. The risk of potential contamination of the food supply is one associated with all genetically enhanced organisms, not just the food crops. Indeed, food supply contamination can occur with nongenetically modified or natural production systems as well!

Although plant-produced pharmaceuticals have raised concern in political debates, it is important that Nature Biotechnology reports science-based facts and arguments and does not give credence to the whims of special interest groups whose position is not science based. Our discussion here should be focused on a safe food supply, and on appropriate safety and containment issues regarding the cost-effective production of pharmaceuticals. A. thaliana, flax or duckweed may be appropriate choices for production of certain plant-made pharmaceuticals, but in other cases it may be corn, potatoes, tomatoes, bananas, soybeans, rice or some other food crop that makes the most sense both scientifically and economically.

\section{Gregory C Phillips}

Arkansas State University, College of Agriculture, PO Box 1080, 119 S. Caraway Road, State

University, Arkansas 72467-1080, USA. e-mail: gphillips@astate.edu

\title{
ELSI and bioterrorism countermeasures?
}

\author{
To the editor: \\ As outlined in a news item in your March \\ issue (Nat. Biotechnol. 22, 256-267, 2004), \\ the United States' proposed appropriations \\ of billions of dollars for bioterror \\ preparedness in 2005 and, presumably, \\ beyond, constitute a scientific and \\ sociopolitical endeavor unprecedented in \\ scope. The only other undertaking that has \\ approached this magnitude as "a major
}

federal R\&D priority" in recent history was the Human Genome Project (HGP). In contrast to the present initiative, however, it was recognized immediately that the HGP had ethical, legal and social implications (ELSI) that needed to be the focus of concerted research efforts. With laudable foresight and responsibility, $3 \%-5 \%$ of the annual HGP budget was earmarked for ELSI research from the outset of the project. This begs a very important question: shouldn't a portion of the bioterror preparedness budget be similarly allocated to ELSI research?

\section{Shane K Green}

Institute for Ethics, American Medical Association, 515 North State St., Chicago, Illinois 60610, USA.

e-mail:Shane_Green@ama-assn.org

\section{Preprocessing of oligonucleotide array data}

\section{To the editor:}

A paper by Zhang et al. ${ }^{1}$ in the July 2003 issue of Nature Biotechnology presents PerfectMatch, a physical-model-based algorithm for modeling the binding interactions between probes on Affymetrix (Santa Clara, CA, USA) GeneChip oligonucleotide arrays and their target nucleic acids. Stochastic-model-based algorithms have been shown to improve the preprocessing of array data to take into account the presence of optical noise, nonspecific binding and probe-specific effects. Although Zhang et al. demonstrate the superior performance of PerfectMatch over two widely used algorithms, MAS 5.0 and dChip, they fail to consider a leading stochastic-model-based procedure, robust multi-array analysis (RMA), from our group $^{2}$. Here, we provide evidence that (i) measurements obtained using RMA are as precise and more accurate than those obtained using PerfectMatch and (ii) the accuracy of stochastic-model-based

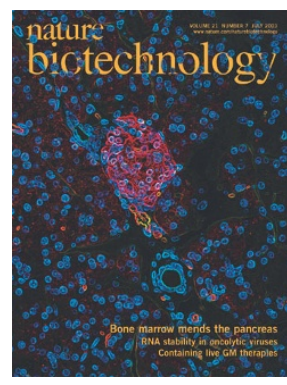
procedures can be improved by carefully incorporating physical model information.

GeneChip arrays are widely used for quantitative and highly parallel measurements of gene expression. Genes are represented on these arrays by pairs of 25-base oligonucleotide probes ${ }^{3}$ denoted probe sets. The first component of these pairs is referred to as a perfect match (PM) probe and is designed to be specific to transcripts from the intended gene. However, background noise and measurement error are unavoidable. Affymetrix's background adjustment approach is to pair each PM with a mismatch (MM) probe designed by changing the middle $\left(13^{\text {th }}\right)$ base with the intention of measuring only background noise and considering the difference PMMM. To obtain an expression measure, their default expression measure (MAS 5.0) is a robust average of log (PM-MM) values ${ }^{4}$. A version of the additive-backgroundmultiplicative-error stochastic model ${ }^{2,5}$ has been demonstrated to describe the behavior of probe-level data well ${ }^{6}$. We refer to this model as the Wu-Irizarry (WI) model. This stochastic model suggests that MAS 5.0 is quite accurate, but that its accuracy is overwhelmed by its impreciseness. These theoretical results have been demonstrated empirically ${ }^{7}$. RMA uses a global background adjustment based on a PM-only estimation procedure designed to avoid MAS 5.0's exaggerated variance. Nevertheless, the WI model predicts that RMA's adjustment does not completely remove nonspecific binding therefore introducing some bias. However, the sacrifice in accuracy is well worth the large gains in precision, resulting in a superior expression measure ${ }^{7}$. 